\title{
Conjugate heat transfer for turbulent flow in a thick walled plain pipe
}

\author{
Eyub Canli ${ }^{1, *}$, Ali Ates ${ }^{1}$, and Sefik Bilir $^{1}$ \\ ${ }^{1}$ Selcuk University, Mechanical Engineering Department, P.K. 42003 Selcuklu Konya, Turkey
}

\begin{abstract}
Laminar and turbulent flow have their own characteristics in respect of heat transfer in pipes. While conjugate heat transfer is a major concern for a thick walled pipe with laminar flow inside it, there are limited studies about a turbulent flow in a thick walled plain pipe considering the conjugate heat transfer. In order to conduct such a work by means of in-house developed code, it was desired to make a preliminary investigation with commercially available CFD codes. ANSYS CFD was selected as the tool since it has a positive reputation in the literature for reliability. Defined heat transfer problem was solved with SIMPLE and Coupled Schemes for pressure velocity coupling and results are presented accordingly.
\end{abstract}

\section{Introduction}

Certain cases of heat transfer involve thick walled pipes and flow inside them. For such a case, not only heat convection transfers heat but also effect of conduction can be important for solid side and may be for the fluid side. This event is called conjugated heat transfer and has been subject of numerical and computational analysis efforts for several decades. Although there are vast amount of literature, some recent works are summarized below.

Iida et al. utilized Direct Numerical Simulation (DNS) for a turbulent channel flow under stable density difference [1]. Authors compared DNS results with experimental results since the study evaluates DNS as a tool and reported that the Nusselt Number $(\mathrm{Nu})$ results agree with each other. Authors identified Internal Gravity Waves as being formed in the core region of the flow. These waves are followed by steep turbulent heat flux suppression and gradients of velocity and temperatures. As a novel output, they reported that the density stratification makes the flow laminar on one wall while the flow on the outher wall remains turbulent [1].

Varol et al. investigated conjugate heat transfer for different vertical walls and natural convection in them [2]. Authors specifically focused on the entropy generation due to the conjugate heat transfer. Entropy generation due to heat transfer is reported to be higher than that of fluid flow irreversibility [2].

Conjugate heat transfer for natural convection seems more popular recently than the forced convection. Kuznetsov and Sheremet [3] conducted a numerical study in order to analyze turbulent natural convection in terms of conjugate heat transfer due to thick wall enclosure. They deliberately defined a relatively complex thermal case. Standard $k-\epsilon$ was used with wall functions. As a different parameter, Grashof number between $10^{8}$ and $10^{10}$ was taken as one of the parameters. They presented very similar results to some examples of the literature in order to validate their work. Then investigated the effects of parameters they choose. Finally they present a correlation of $\mathrm{Nu}$ against Grashof Number [3].

Similar to the natural convection for conjugate heat transfer, another trending research field is on DNS of the conjugate heat transfer. DNS of turbulent channel flow with conjugate heat transfer at Prandtl Number (Pr) 0.01 is reported by Tiselj and Cizelj [4]. Additionally fluctuating temperature boundary condition was investigated alongside with the non-fluctuating temperature boundary condition. The selected $\mathrm{Pr}$ corresponds to the liquid sodium-steel contact. It is indicated that the turbulent temperature fluctuations can affect temperature distribution of the wall [4].

The most relevant work to the target investigation of the authors of the present paper is reported by Yahya et al. [5]. They utilized Thermal Large Eddy Simulation (TLES). Since this model only models sub grid eddies, more realistic flow structures can be attained and authors visualized their results in a successful manner in this respect. They considered effect of changing viscosity. As a main result they found that the turbulent kinetic energy decreases near the hot wall as the viscosity of the fluid increases and cold wall enhances the turbulence [5]. A similar work is presented by Satish and Venkatasubbaiah [6]. The flow is enclosed between two flat plates but with a significant difference; one of the plates is moving. They reported that heat conduction through the wall changes the heat transfer characteristics significantly. Actually their major findings are expected phenomena however the addition of heat transfer enhancement by means of increasing moving plate speed is important. They also used $k$ - $\epsilon$ turbulence model [6].

Corresponding author: ecanli@selcuk.edu.tr 
Analytical solution of conjugate turbulent forced convection boundary layer flow over plates is presented by Shariatzadeh [7]. A semi-analytical solution based on differential transform method is presented in the paper. The method is for solving the non-linear differential equation in the problem. The author indicates that a slight temperature difference occurs in conjugate case comparing to the non-conjugate case.

Conjugate heat transfer along a heated flat plate is analyzed by using wall resolved LES [8]. The authors indicate that a locally dynamic Smagorinsky SGS model was implemented into the LES solver to enable the backscatter phenomenon intrinsic to transitioning boundary layer flows for the subgrid scale closure. This kind of LES is reported to be convenient considering the response capability and experimental results [8].

Laminar-turbulent transition in case of a conjugate heat transfer scenario is reported for assessing RANS [9]. Performance of totally 8 different turbulence model are given. They also add some other parameters to the study such as inclination angle and Richardson number and investigate the location of the transition point.

This work presents temperature distribution results of ANSYS CFD for a thick walled plain circular pipe and turbulent flow inside it. The results will also be used as a comparison tool for an ongoing $\mathrm{PhD}$ work.

\section{Method}

A $2 \mathrm{~m}$ plain pipe having a thick wall and turbulent flow inside it are considered for a conjugated heat transfer case. Computational Fluid Dynamics (CFD) was chosen as the tool of investigation. Since there is an ongoing work to develop an in-house code, it is desired to obtain preliminary results from commercial codes for comparison and also mention about the ongoing work. Therefore ANSYS software was used for all the CFD steps. The two dimensional, axis-symmetrical flow domain was created for two different dimensions in ANSYS Space Claim module. Figure 1 shows the two drawings. The only difference between flow domains are the wall thickness. The first flow domain is $0.01 \mathrm{~m}$ thick and the second one is $0.025 \mathrm{~m}$ thick. Flow length is $2 \mathrm{~m}$ and the pipe radius is $0.05 \mathrm{~m}$. Although materials can be selected in the solver, wall material was selected as 405 stainless steel and fluid was selected as water at room temperatures. Naming edges and domains and relating edges with each other were left to mesh generator.

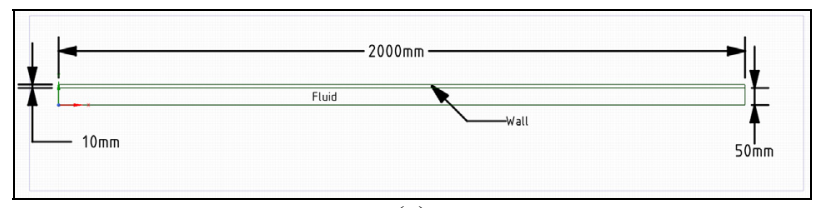

(a)

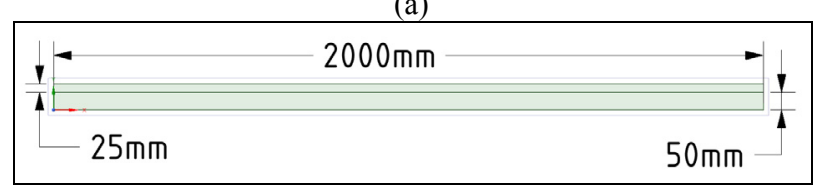

(b)

Fig.1. The dimensions of the 2D axis-symmetric flow domains in ANSYS Space Claim
Mesh was generated in the ANSYS Mesh module. Two different mesh files were generated due to the two different thickness of the wall. However the mesh element number of the flow domain remained same. The proper mesh number for the flow domain was found to be 80,000 elements and reported in "Comparison of turbulence models and solution options for a thick walled plain pipe" titled conference paper [10] in the present conference. Therefore same structured mesh was arranged for the flow domain. $0.05 \mathrm{~m}$ pipe radius was divided into 100 units with a bias factor of 10 . The thinnest cell is next to the wall and the widest cell is next to the symmetry axis. $2 \mathrm{~m}$ pipe length was divided 800 units with again a bias factor of 10 . Therefore the flow domain mesh exhibits a view that it is stretched towards the outlet of the pipe and the symmetry axis. These arrangements were done by using 4 edge sizing and 1 face mesh options. All the sizing arrangements were set to "hard" behavior meaning that they forced the sizes by setting the edge sizes at the beginning of mesh generation incident. Wall edges parallel to the symmetry axis were divided similar to the pipe due to assure that the cells correspond to each other perfectly. The two side edges of the wall were divided into 10 uniform pieces for $0.01 \mathrm{~m}$ wall thickness and 25 uniform pieces for $0.025 \mathrm{~m}$ wall thickness. All edges and faces are named in the mesh generator. The south edge of the wall and the north edge of the fluid according to view plain were set as contact region. Figure 2 shows the inlet and outlet views of the meshes. Figure 3 is for the edge names and Figure 4 shows the contact region.

According to the mesh report, the geometry is fully defined. Although the drawing and the solution is two dimensional, the mesh generator always puts a unit length to the third dimension. Therefore a volume value is seen equal to the surface area of the analysis domain. For the $0.01 \mathrm{~m}$ wall thickness case, there is $0.12 \mathrm{~m}^{2}$ surface area therefore $0.12 \mathrm{~m}^{3}$ volume can be seen in the mesh report. 85,707 nodes and 84,000 elements exist for $0.01 \mathrm{~m}$ wall thickness and 101,727 nodes and 100,000 elements exist for $0.025 \mathrm{~m}$ wall thickness. Element quality values are min. 0.04, max. 0.99, av. 0.46. However these element quality values are better for the wall side because of the uniform distribution of the cells. The wall side element quality values are min. 0.48 , max. 0.99 , av. 0.82. Preferred physics option was set to CFD and solver preference was set to Fluent. Remaining settings were leaved as default and no inflation was arranged since the near wall region has sufficient mesh elements in it. This is ensured by checking wall $\mathrm{y}^{+}$ values. 


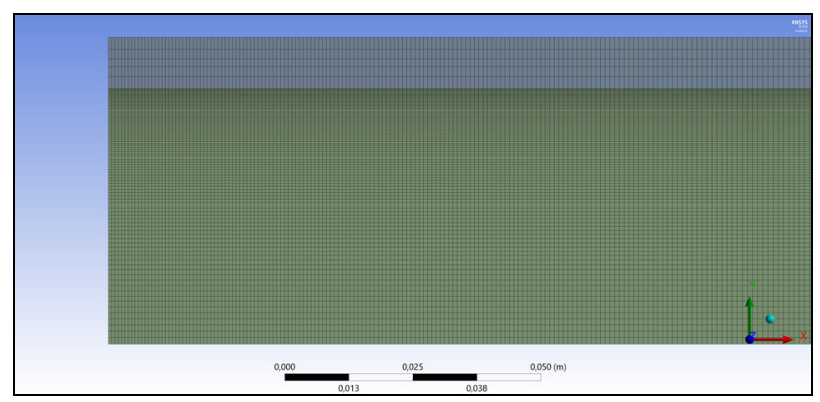

(a)

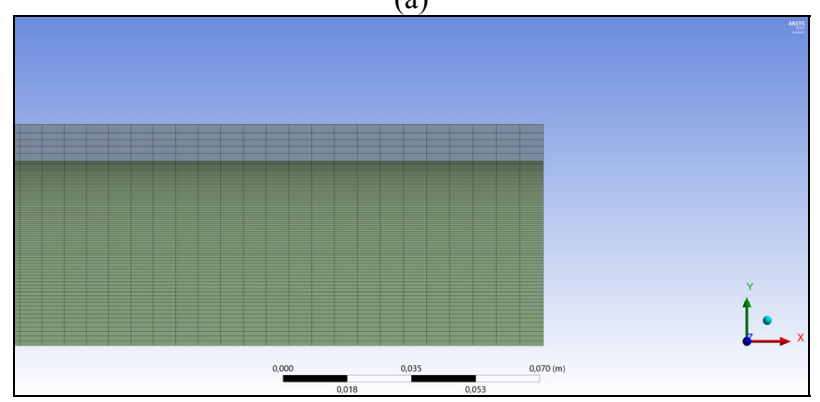

(b)

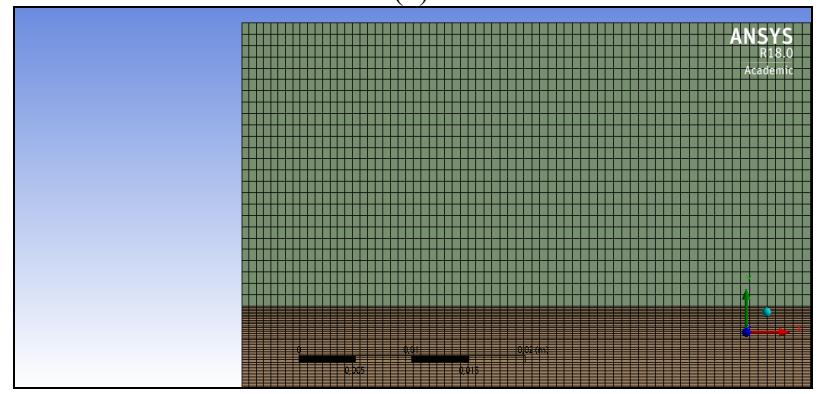

(c)

Fig.2. Mesh views (a) inlet of the $0.01 \mathrm{~m}$ wall pipe (b) outlet of the $0.01 \mathrm{~m}$ wall pipe (c) inlet of the $0.025 \mathrm{~m}$ wall pipe

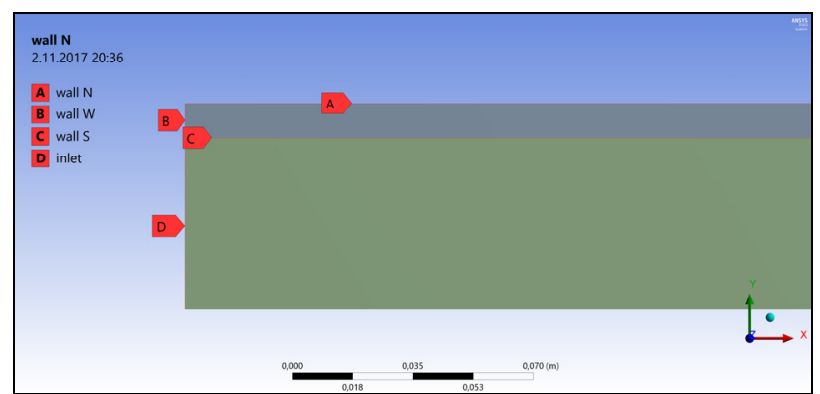

(a)

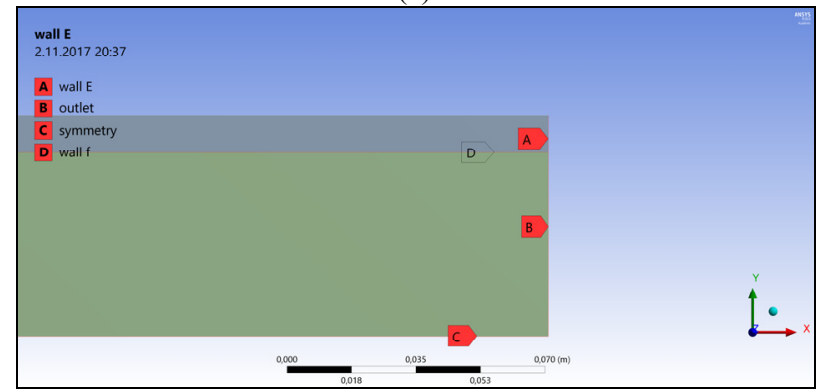

(b)

Fig.3. Names of the boundaries (a) inlet side (b) outlet side

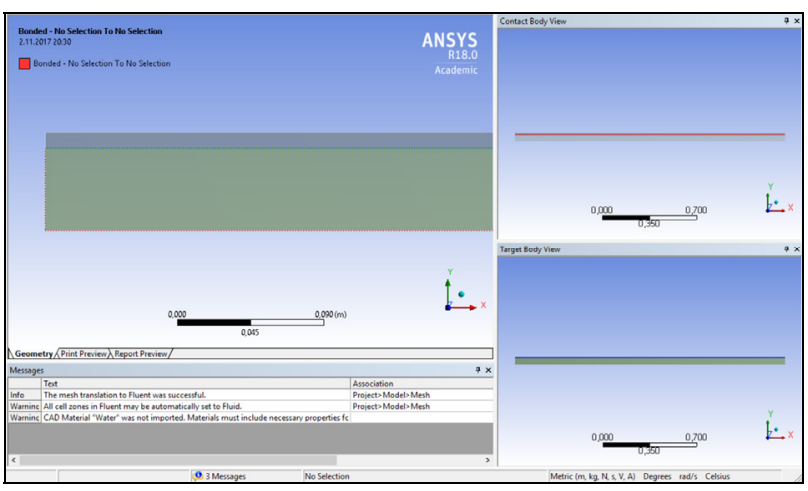

Fig.4. The contact region between solid and fluid domains in mesh generator

Fluent Solver initial setup settings are: single precision, serial processing, 2D. The general settings for the solver are pressure based, absolute velocity formulation, steady and 2D axis-symmetric. Effect of the gravity was ignored. Standard $k-\epsilon$ with standard wall function was selected as the turbulence model alongside with the energy equation. Fluid material was selected as liquid water and wall material was selected as steel. Thermodynamic properties were selected as constant. Density of the water was $998.2 \mathrm{~kg} / \mathrm{m}^{3}$ and steel was 8030 $\mathrm{kg} / \mathrm{m}^{3}$. Specific heat values were 4182 and $502.48 \mathrm{j} / \mathrm{kg} \cdot \mathrm{K}$ for water and steel respectfully. Water had $0.6 \mathrm{~W} / \mathrm{m} \cdot \mathrm{K}$ thermal conductivity while steel had $16.27 \mathrm{~W} / \mathrm{m} \cdot \mathrm{K}$. Water viscosity was $0.001003 \mathrm{~kg} / \mathrm{m} \cdot \mathrm{s}$. Water inlet was set to $0.08937 \mathrm{~m} / \mathrm{s}$ absolute velocity magnitude that corresponds to 10,000 Reynolds Number (Re) while initial turbulence intensity was set to $5 \%$ and hydraulic diameter was set to $0.1 \mathrm{~m}$. The inlet water temperature was $300 \mathrm{~K}$. Outlet boundary condition was selected as 0 Pa gauge pressure. All walls were set as wall boundary condition with $0 \mathrm{~W} / \mathrm{m}^{2}$ constant heat flux except for the north edge of the wall. North edge of the wall set to 400 $\mathrm{K}$ and $500 \mathrm{~K}$ for separate calculations. Symmetry axis was set to symmetry boundary condition and connection edges were similarly set as connection boundary condition. Reference values were taken from the water inlet. Two different solution schemes were used; namely SIMPLE and Coupled. Therefore it can be said that three parameters were changed during analysis; wall thickness, pipe outside surface temperature and solution scheme. First of all, the flow dynamics obtained by SIMPLE and Coupled schemes are compared and then SIMPLE scheme was used for different temperature and thickness values. The point values comparison of the SIMPLE and Coupled schemes are given in Table 1.

Table 1. The point values comparison of the SIMPLE and Coupled schemes

\begin{tabular}{|c|c|c|}
\cline { 2 - 3 } \multicolumn{1}{c|}{} & SIMPLE & Coupled \\
\hline Vel. (m/s) ) at symmetry outlet & 0.105 & $0.119(13 \%)$ \\
\hline Tot. Pres. (Pa) ) at symmetry outlet & 5.48 & $7.07(29 \%)$ \\
\hline Turb. Int. ) at symmetry outlet & 0.045 & $0.054(20 \%)$ \\
\hline Turb. Vis. (Pa*s) ) at symmetry outlet & 0.0275 & $0.03154(14 \%)$ \\
\hline y+ at connection outlet & 0.587 & $0.56(4.5 \%)$ \\
\hline Temperature (K) at symmetry outlet & 305.557 & $309.258(1.2 \%)$ \\
\hline Temperature (K) at connection outlet & 368.929 & $366.253(0.7 \%)$ \\
\hline Av. \% Dif. & - & $11.7 \%$ \\
\hline
\end{tabular}


The spatial discretization options were left as default and they were Least Squares Cell Based Gradient; Second Order Pressure; Second Order Upwind Momentum; First Order Upwind Turbulent Kinetic Energy; First Order Upwind Turbulent Dissipation Rate; Second Order Upwind Energy. Solution control under relaxation factors were selected as 0.3 for pressure, 1 for density, 1 for body forces, 0.7 for momentum, 0.8 for turbulent kinetic energy, 0.8 for turbulent dissipation rate, 1 for turbulent viscosity, 1 for energy. Hybrid initialization was used. Solution was converged about 100 iterations in minutes.

CFD Post was used for post processing. The computational domain is seen three dimensional though the design is two dimensional. This is thought to be due to the infrastructure of the ANSYS CFD software when axis-symmetrical solution is used. The $3 \mathrm{D}$ view of the computational domain is given in Figure 5.

Since the aspect ratio of the domain is very small (height/length $=0.03$ ), $\mathrm{x}-\mathrm{y}$ plots are utilized for the results mostly. Only close inlet and outlet contour views of the temperature are given as graphical results.

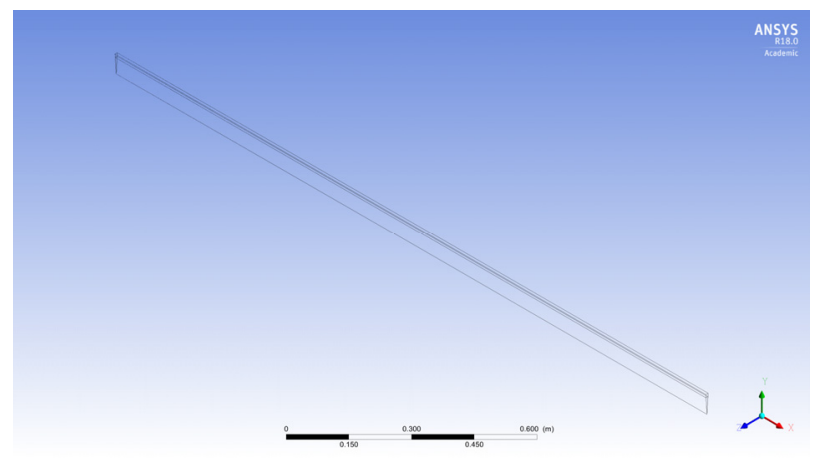

(a)

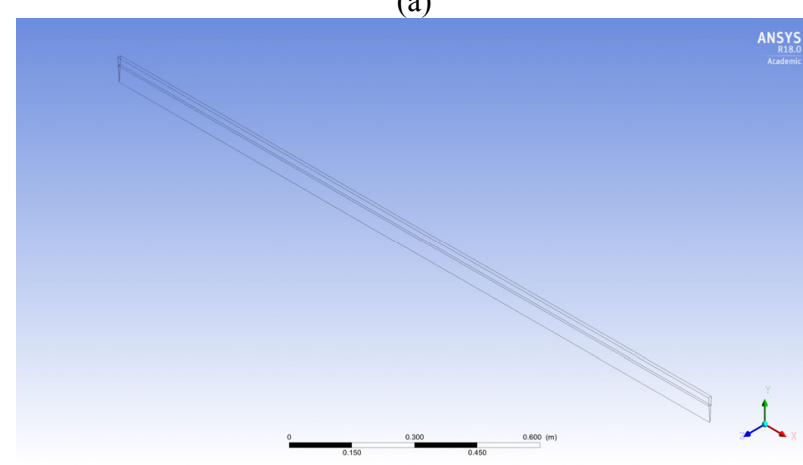

(b)

Fig.5. 3D CFD Post views (a) $0.01 \mathrm{~m}$ wall thickness (b) 0.025 wall thickness

\section{Results}

Initially comparison of SIMPLE scheme with Coupled scheme for pressure velocity coupling is given in terms of velocity, wall $\mathrm{y}^{+}$, turbulence intensity and viscosity in Figure 6.

The inlet fluid temperature contours of two different wall thicknesses and two different wall outside temperature boundary conditions are given in Figure 7. Similarly inlet wall temperature contours are given in Figure 8 . The outlet fluid temperature contours of two different wall thicknesses and two different wall outside temperature boundary conditions are given in Figure 9. And finally outlet wall temperature contours are presented in Figure 10.

The pressure velocity coupling by Coupled Scheme shows smoother flow values comparing to SIMPLE scheme, however the $u-y$ velocity profiles of the Coupled Scheme show that the flow develops later. Additionally wall $\mathrm{y}+$ values are lower for Coupled scheme. The turbulent viscosity and intensity values of the Coupled Scheme indicate that this scheme produces additional turbulence information however the temperature profiles of the two schemes are very similar. Only $T-y$ profiles of the Coupler Scheme show greater distribution of the temperature. According to the present results, it is decided that the less work burden scheme can be chosen for the present case.

Temperature profiles show that the thermal development is not acquired with the current pipe length. This can be evaluated with a dimensionless analysis with a semi-infinite pipe length through downstream. It can also be inferred that a transient analysis depending on time can yield different temperature profiles for a period of time and then the temperature profiles change with location.

Temperature contours tended identical for different wall outside temperatures since the thermodynamic properties were taken constant. The change of wall thickness has an effect only on magnitudes similar to the wall outside thickness.

\section{Conclusions}

This work is a preliminary study surveying the results of a commercial CFD code for comparison with an inhouse developed code while assessing some fundamental settings and methods in CFD for the investigated case. A thick walled plain pipe is analyzed with $2 \mathrm{D}$ axissymmetrical design for turbulent flow of $R e=10,000$ and heat transfer at constant outside wall temperature. The interface condition is unknown and obtained as a result. Results of SIMPLE and Coupled pressure velocity coupling schemes are compared. Following remarks can be noted.

- Pressure velocity coupling avoids velocity anomalies of the $k-\epsilon$ with the present structured mesh. The low element quality at the medium and far downstream region of the fluid domain may cause these anomalies with SIMPLE Scheme. Therefore Coupled Scheme can give more information about the turbulence for the present case and setup.

- When thermodynamic properties are taken constant, changing value of the constant outside surface temperature and wall thickness only changes the magnitudes but the behavior or the tendency remain same for the steady calculation. Transient solution may yield different results according to time. 

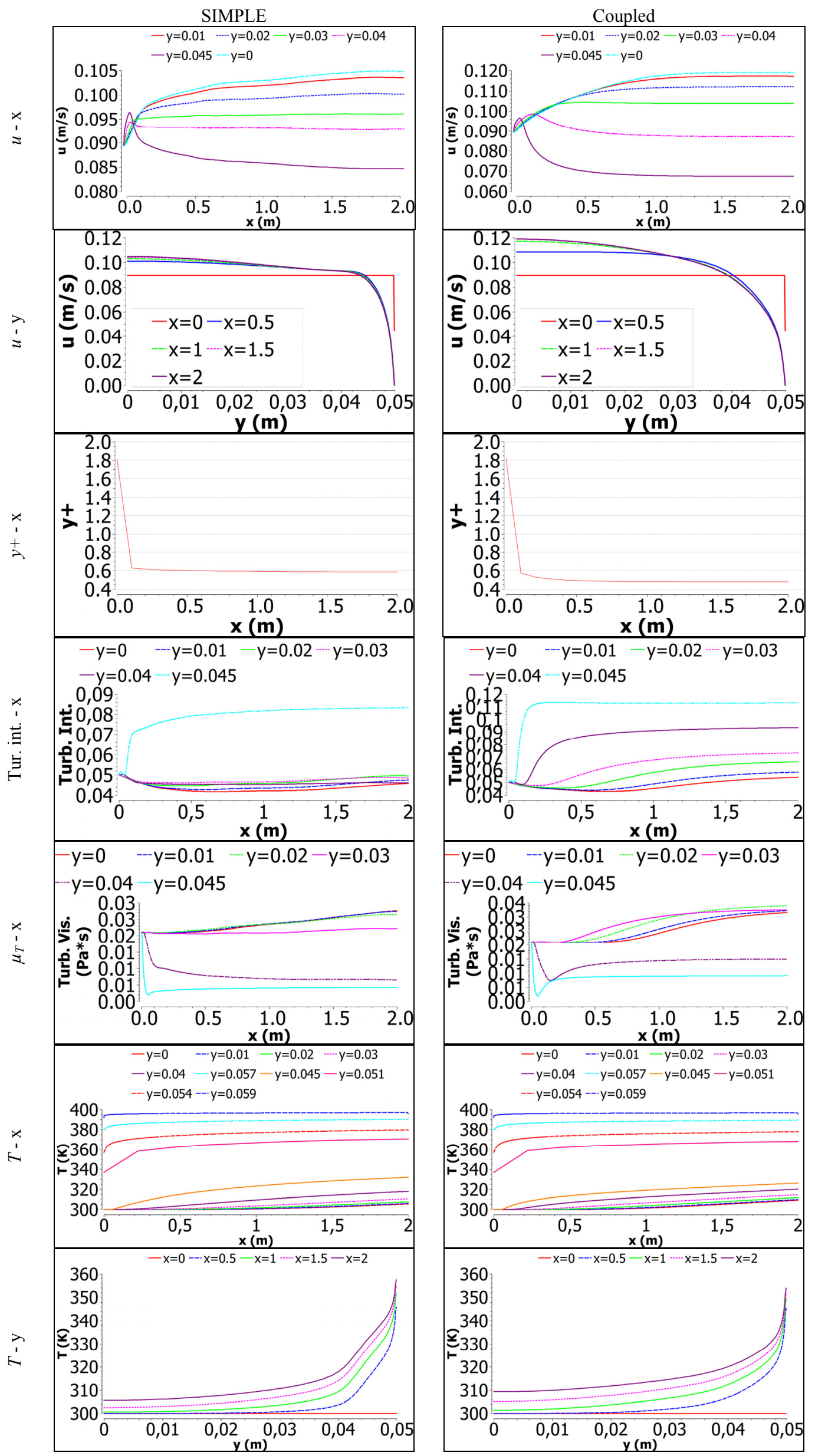
- Conjugate heat transfer cannot be identified in the present turbulent flow with characteristics similar to the laminar flow since the thermal boundary condition is applied at the beginning of the pipe and lasts till the end. The steady simulation and relatively fast flow also avoided effects of axial heat conduction of the fluid.

In the future, authors are planning to make a parametrical study for conjugated heat transfer in a circular thick walled pipe while turbulent flow exists inside it by constituting and using an in-house developed code. Not only physics will be investigated but also numerical and computational approach will be tried to be improved.

\section{Acknowledgment}

The academic license of the software used in this work is belonged to Selcuk Univertsity. Authors also would like to acknowledge the financial support of Selcuk University Scientific Research Projects Coordination Unit for the conference participation (project no: 17701387).

\section{References}

1. O. Iida, N. Kasagi, Y. Nagano, International Journal of Heat and Mass Transfer 45, (2002)

2. Y. Varol, H. F. Oztop, A. Koca, International Communications in Heat and Mass Transfer 35, (2008)

3. G. V. Kuznetsov, M. A. Sheremet, International Journal of Heat and Mass Transfer 53, (2010)

4. I. Tiselj, L. Cizelj, Nuclear Engineering and Design 253, (2012)

5. S. M. Yahya, S. F. Anwer, S. Sanghi, International Journal of Thermal Sciences 88, (2015)

6. N. Satish, K. Venkatasubbaiah, Applied Thermal Engineering 100, (2016)

7. O. J. Shariatzadeh, Thermal Science 20,5, (2016)

8. A. Ojofeitimi, Y. Hattori, International Journal of Heat and Mass Transfer 109, (2017)

9. M. Abdollahzadeh, M. Esmaeilpour, R. Vizinho, A. Younesi, J.C. Pàscoa, International Journal of Heat and Mass Transfer 115, (2017)

10. E. Canli, A. Ates, S. Bilir, Experimental Fluid Mechanics 2017 (EFM 2017) (to be published in the proceedings) 


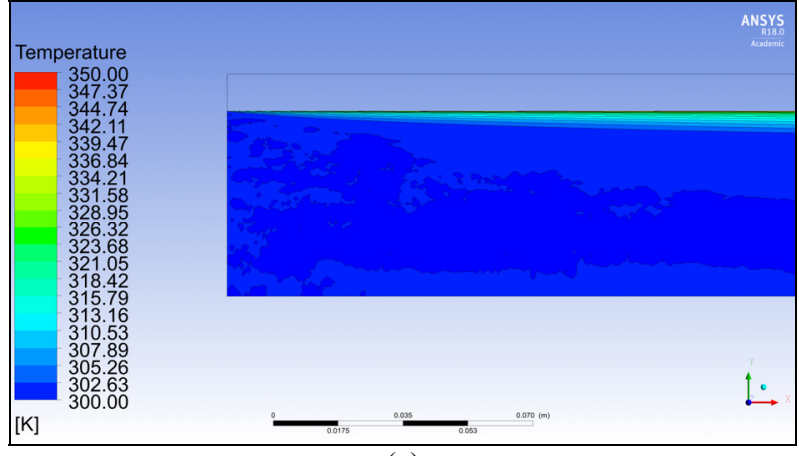

(a)

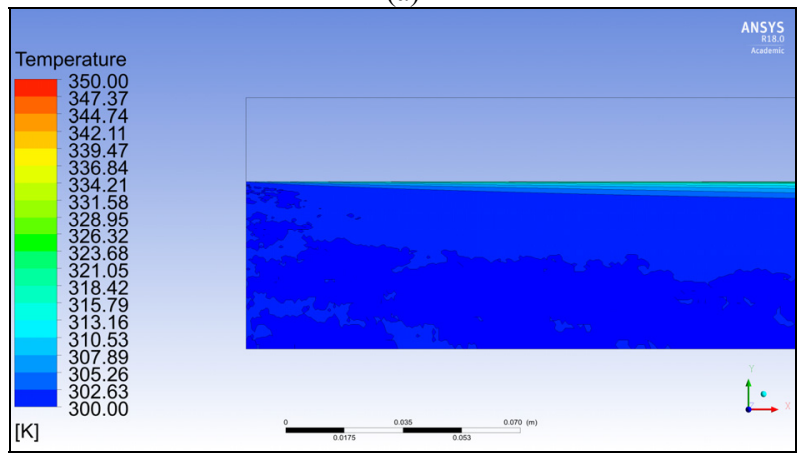

(c)

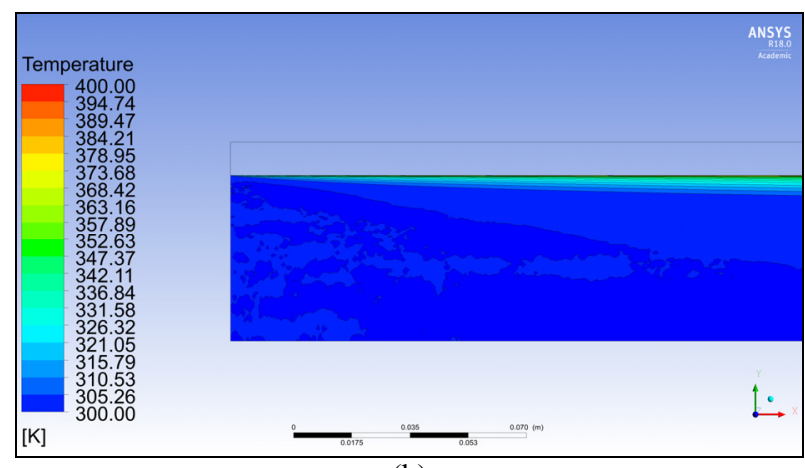

(b)

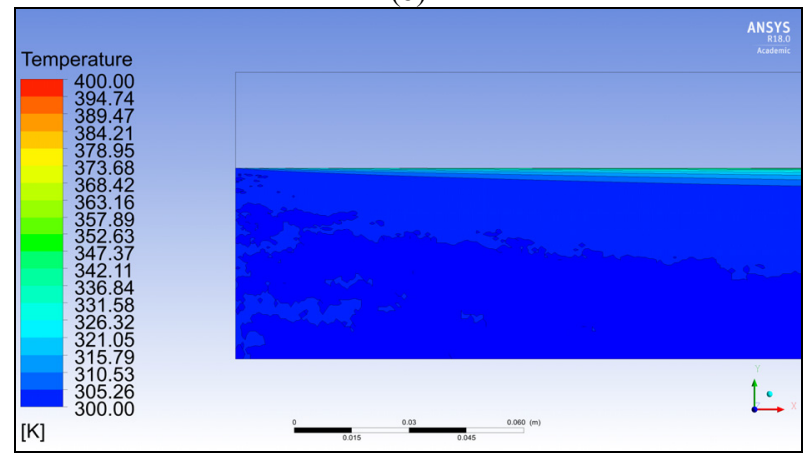

(d)

Fig.7. Inlet fluid temperature contours (a) $0.01 \mathrm{~m}$ wall thickness \& $400 \mathrm{~K}$ wall outside temperature (b) $0.01 \mathrm{~m}$ wall thickness \& 500 $\mathrm{K}$ wall outside temperature (c) $0.025 \mathrm{~m}$ wall thickness \& $400 \mathrm{~K}$ wall outside temperature (d) $0.025 \mathrm{~m}$ wall thickness \& $500 \mathrm{~K}$ wall outside temperature

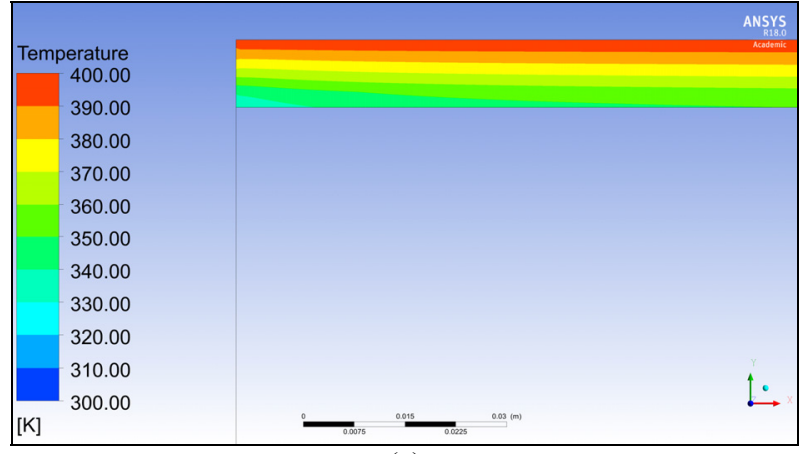

(a)

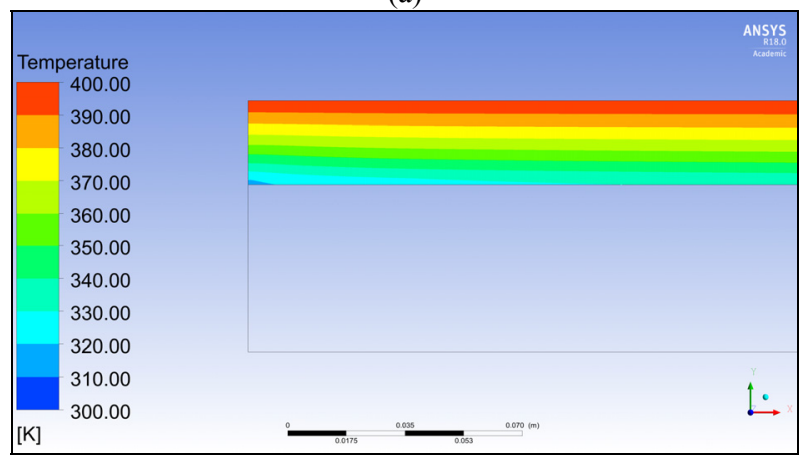

(c)

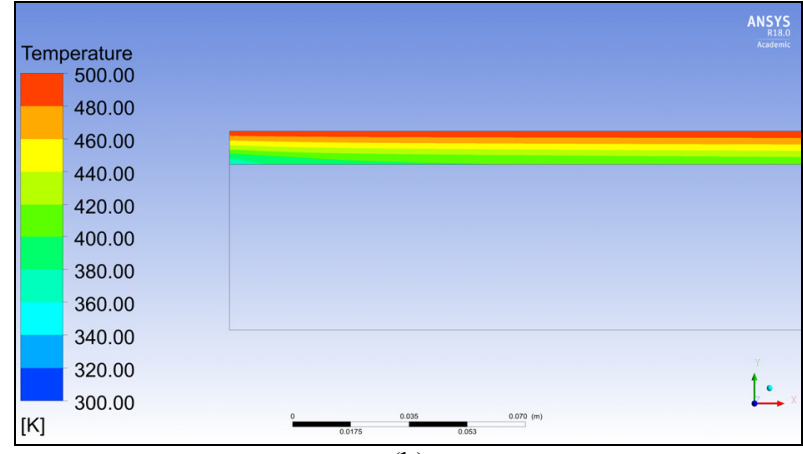

(b)

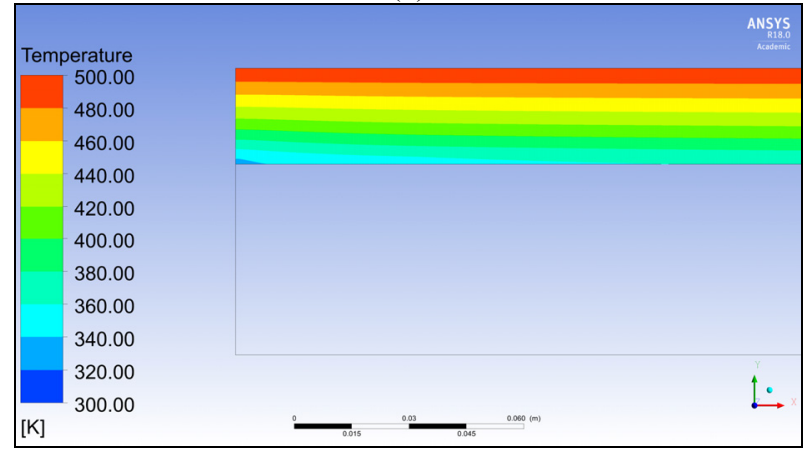

(d)

Fig.8. Inlet wall temperature contours (a) $0.01 \mathrm{~m}$ wall thickness \& $400 \mathrm{~K}$ wall outside temperature (b) $0.01 \mathrm{~m}$ wall thickness \& 500 $\mathrm{K}$ wall outside temperature (c) $0.025 \mathrm{~m}$ wall thickness \& $400 \mathrm{~K}$ wall outside temperature (d) $0.025 \mathrm{~m}$ wall thickness \& $500 \mathrm{~K}$ wall outside temperature 


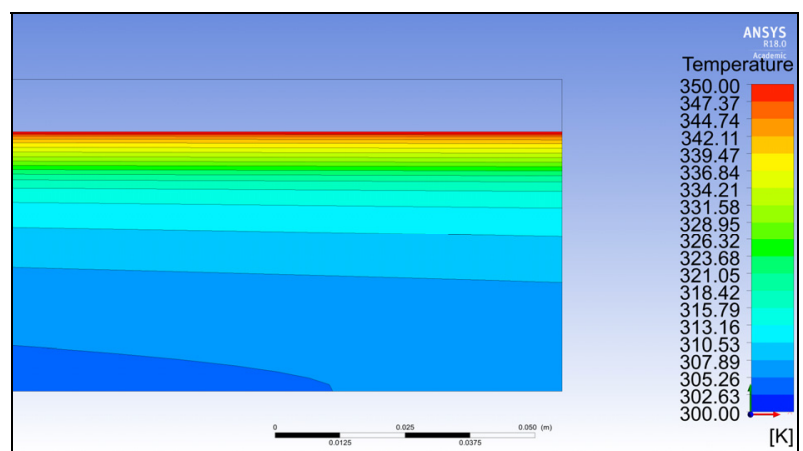

(a)

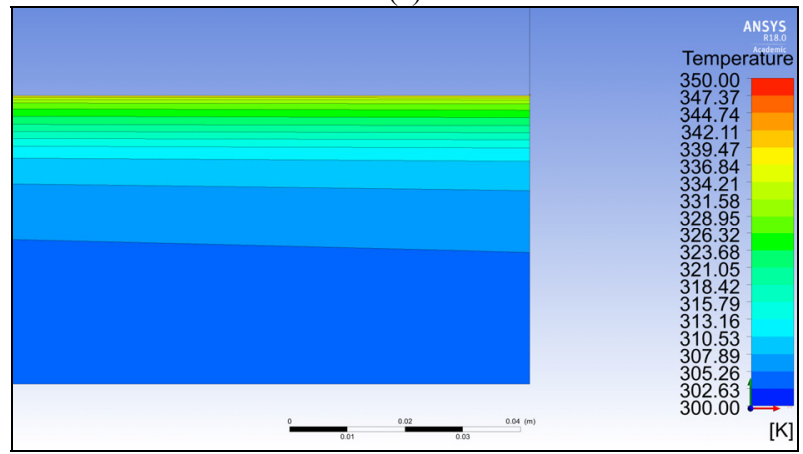

(c)

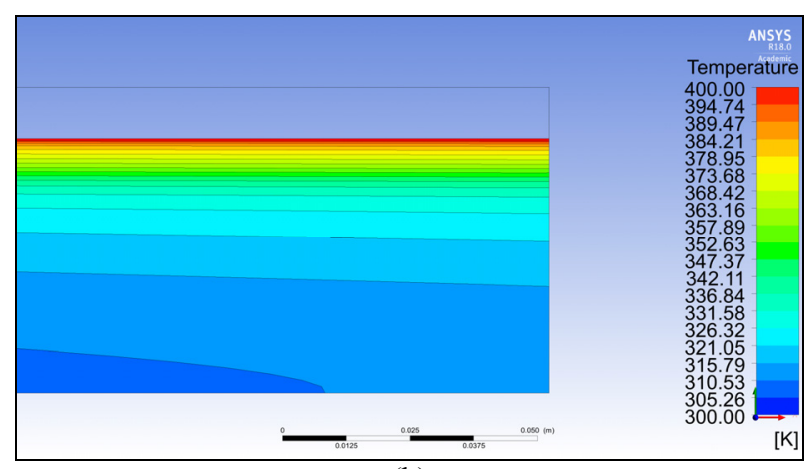

(b)

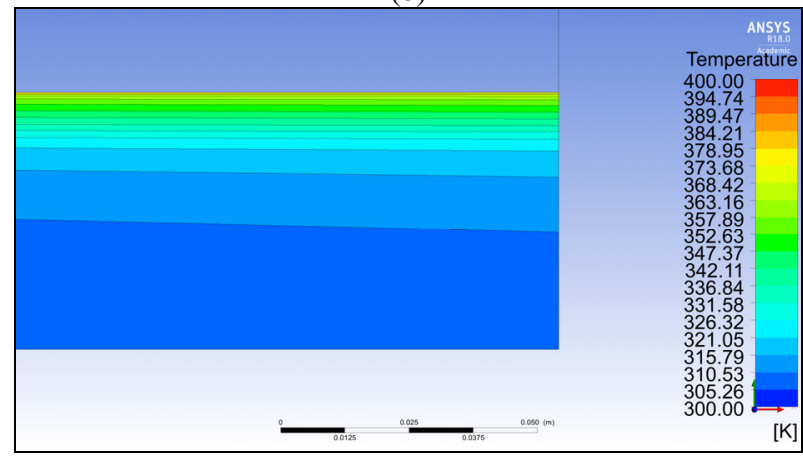

(d)

Fig.9. Outlet fluid temperature contours (a) $0.01 \mathrm{~m}$ wall thickness \& $400 \mathrm{~K}$ wall outside temperature (b) $0.01 \mathrm{~m}$ wall thickness \& $500 \mathrm{~K}$ wall outside temperature (c) $0.025 \mathrm{~m}$ wall thickness \& $400 \mathrm{~K}$ wall outside temperature (d) $0.025 \mathrm{~m}$ wall thickness \& $500 \mathrm{~K}$ wall outside temperature

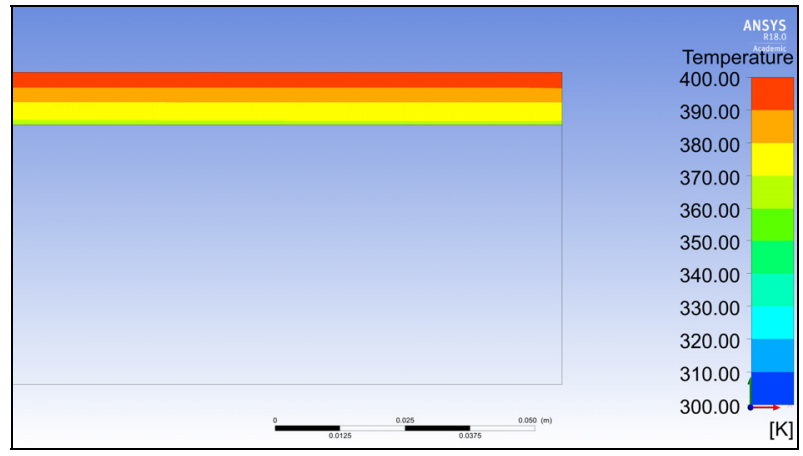

(a)

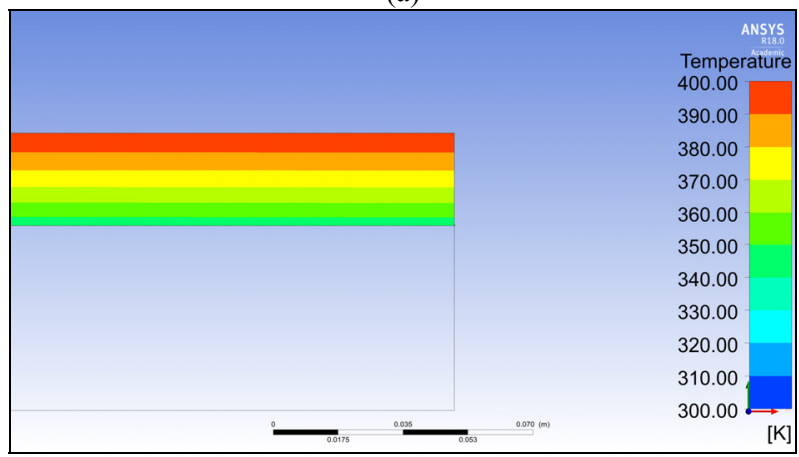

(c)

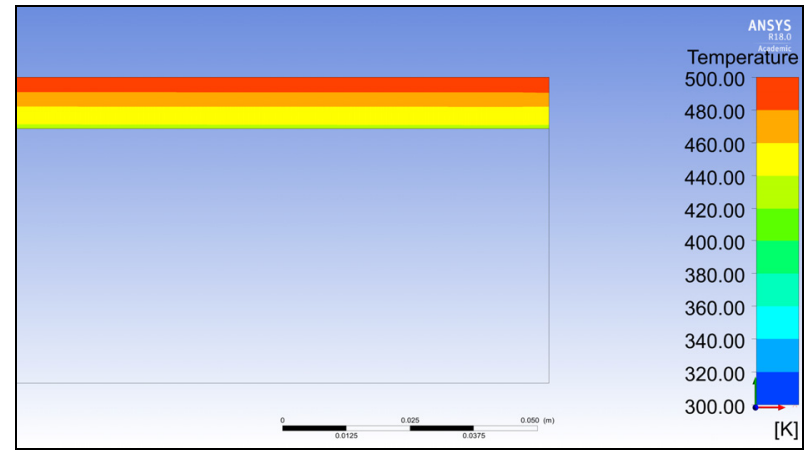

(b)

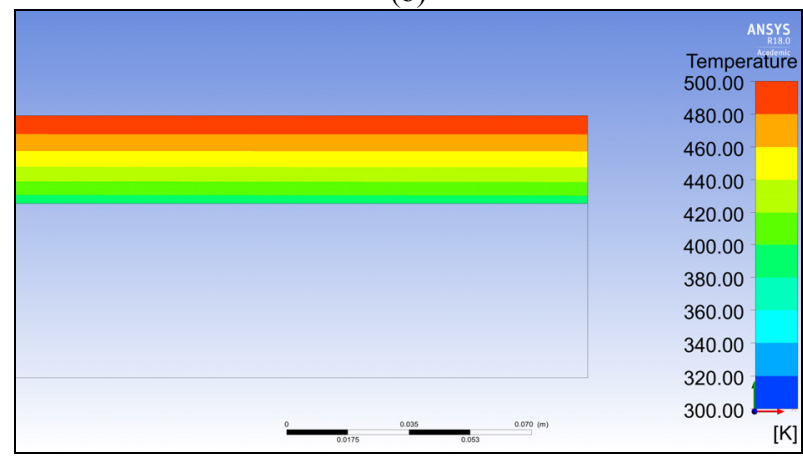

(d)

Fig.10. Outlet wall temperature contours (a) $0.01 \mathrm{~m}$ wall thickness \& $400 \mathrm{~K}$ wall outside temperature (b) $0.01 \mathrm{~m}$ wall thickness \& $500 \mathrm{~K}$ wall outside temperature (c) $0.025 \mathrm{~m}$ wall thickness \& $400 \mathrm{~K}$ wall outside temperature (d) $0.025 \mathrm{~m}$ wall thickness \& $500 \mathrm{~K}$ wall outside temperature 